\title{
ESTRATÉGIAS DE ENFRENTAMENTO UTILIZADAS POR PSICÓLOGOS: UMA ANÁLISE DE GÊNERO
} COPING IN PYCHOLOGISTS: A GENDER ANALYSIS

\author{
SANDRA YVONNE SPIENDLER RODRIGUEZ 1; MARY SANDRA CARLOTTO 2; MARIANA BARCINSKI 3 \\ (FACULDADE DE DESENVOLVIMENTO DO RIO GRANDE DO SUL, Porto Alegre /RS, Brasil)
}

\begin{abstract}
RESUMO
OBJETIV0: avaliar as estratégias de enfrentamento utilizadas por psicólogos frente a estressores ocupacionais e identificar diferenças de acordo com 0 sexo. METODO: A amostra não probabilística constituiu-se de 518 participantes. Foram utilizados como instrumentos de pesquisa a Escala COPE - Estratégias de Enfrentamento e um questionário para levantamento de dados sociodemográficos. RESULTADOS: Os resultados obtidos por meio da prova $\dagger$ de student, considerando a variável sexo, revelaram que as mulheres apresentaram médias mais elevadas nas estratégias focadas na emoção quando comparadas aos homens. CONCLUSÕES: Os resultados apontam para a necessidade de ações diferenciadas para homens e mulheres visando à prevenção de adoecimento ocupacional.
\end{abstract}

PALAVRAS-CHAVES: Estresse ocupacional, estratégias de enfrentamento, gênero, psicólogos.

\section{ABSTRACT}

OBJECTIVE: To evaluate coping strategies dealing with occupational stressors and identify differences according to gender. METHOD: A nonprobability sample composed of 518 informants was analyzed. The COPE Scale, coping and a questionnaire were used as instruments to collect socio-demographic data. RESULTS: Regarding emotion-focused strategies, women registered a higher mean than men. CONCLUSION: Women and men need different type of strategies to prevent occupational stress.

KEY WORDS: Estrés ocupacional, estrategias de enfrentamiento, género, psicólogos

1. Psicóloga, doutora em Psicología. E-mail: psicologa07@gmail.com Endereço para correspondência: Rua Artur Azevedo, 35- Bairro Partenon, Porto Alegre/RS. 2. Psicóloga, doutora em Psicologia Social; PPG Psicologia, Pontifícia Universidade Católica do Rio Grande do Sul, Porto Alegre/RS/Brasil. E-mail: mscarlotto@pesquisador.cnpq.br 3. Psicóloga, doutora em Psicologia do Desenvolvimento; PPG Psicologia, Pontifícia Universidade Católica do Rio Grande do Sul, Porto Alegre/RS/Brasil. E-mail: mariana.barcinski@pucrs.br 


\section{INTRODUÇÃO}

As estratégias de enfrentamento (Coping) têm sido tradicionalmente conceituadas como um conjunto dinâmico de respostas ou reações das pessoas frente a eventos estressores percebidos como uma ameaça ao seu bem-estar psicológico e físico (Carver, Scheier, \& Weintraub, 1989; Folkman, 2009; Leipold \& Greve, 2009). Trata-se de esforços para prevenir ou diminuir a ameaça, dano, perda ou para reduzir o sofrimento associado ao estresse (Carver \& Connor-Smith, 2010). O modelo de Lazarus e Folkman (1984), baseado na avaliação do estressor, sugere que as estratégias de enfrentamento adaptativas podem aumentar os sentimentos de equilíbrio, enquanto as mal adaptativas ocasionam um efeito contrário, sendo associadas a problemas de saúde (Keogh \& Herdenfeldt, 2002).

A forma com as pessoas lidam com os estressores relaciona-se com sua qualidade de vida (Graven \& Grant, 2013) e saúde (Keogh \& Herdenfeldt, 2002). Estratégias centradas na emoção são negativamente associadas com a qualidade de vida física e psicológica, ao passo que as estratégias focalizadas no problema se associam positivamente às mesmas dimensões da qualidade de vida (Graven \& Grant, 2013).

As estratégias utilizadas podem ser focadas no problema, na emoção e na evitação (Tamres, Janicki, \& Helgeson, 2002). Quando a pessoa foca-se no problema, realiza esforços para atenuar 0 estressor para sentir-se melhor (Folkman \& Lazarus, 1988). Ao enfrentar estressores focando-se na emoção, o individuo tenta regular a reação emocional ao evento. 0 foco na esquiva centra-se no envolvimento em atividades ou estados mentais para retirar 0 evento estressante (Lazarus \& Folkman, 1984; Tamres et al., 2002).
Carver, Sheier e Weintraub (1989) desenvolveram, baseados na categorização originalmente proposta por Folkman e Lazarus (1980), um modelo teórico com quinze tipos de estratégias de enfrentamento: a) coping ativo, que consiste no processo de estabelecer passos sucessivos para remover, atenuar ou melhorar os efeitos do estressor; b) planejamento, que implica na atividade de pensar sobre alternativas para lidar com um estressor através da execução de uma ação; c) supressão de atividades concomitantes, que se caracteriza pela eliminação de atividades que distraiam 0 sujeito do foco representado pelo estressor; d) coping moderado, que significa aguardar a oportunidade adequada para efetuar a ação, restringindo a impulsividade; e) busca de suporte social por razões instrumentais, que configura-se pela procura por conselhos ou informações sobre o estressor; f) busca de suporte social por razões emocionais, é considerado a procura de apoio moral, compaixão ou entendimento; g) foco na expressão de emoções, caracterizado pela tendência em focalizar-se na experiência de estresse, expressando sentimentos negativos; $h$ ) desligamento comportamental, entendido pelo abandono das tentativas para atingir metas nas quais esteja presente a interferência do estressor; i) desligamento mental, que ocorre através da utilização de atividades alternativas para afastar 0 problema da mente; j) reinterpretação positiva, que consiste em reinterpretar uma situação negativa ou tensa em termos positivos; k) negação, estratégia na qual ocorre a recusa em acreditar na existência do estressor ou agir como se esse não fosse real; I) aceitação, que corresponde, em um primeiro momento, à percepção do estressor como real e, num segundo, à aceitação do estressor como um fenômeno natural; $\mathrm{m}$ ) religiosidade, entendida como a tendência a voltar-se para a religião como forma de aliviar a tensão; n) humor, que se configura como a estratégia de fazer graça da situação estressora e o) uso de substâncias, que consiste em se desviar do 
problema através do uso de substâncias psicoativas que interfiram na capacidade de avaliação das situações.

As estratégias utilizadas apresentam variações de acordo com o contexto cultural (Heppner, 2008), processo de socialização (Piko, 2001), tipo de profissão (Mazzola, Schonfeld, \& Spector, 2011) e gênero (González-Morales, Peiró, Rodríguez \& Greenglass, 2006; Mazzola, Schonfeld, \& Spector, 2011; Piko, 2001).

Com relação ao gênero, estudos têm demostrado que homens utilizam com maior frequência as estratégias focadas no problema e agem diretamente sobre 0 estressor, enquanto as mulheres geralmente utilizam estratégias focadas na emoção (Keogh \& Herdenfeldt, 2002; NolenHoeksema, 2012; Ptacek, Smith \& Dodge, 1994). As formas de enfrentamento adotadas distintamente por homens e mulheres podem estar vinculadas a questões de gênero que caracterizam determinadas profissões. As profissões socialmente consideradas como femininas são aquelas que envolvem o cuidado, 0 relacionamento interpessoal e a expressão de afeto, aspectos valorizados no processo de socialização das mulheres. Já as consideradas masculinas vinculam-se à autonomia, à competitividade e à racionalidade (Lassance \& Magalhães, 1997).

Os psicólogos estão envolvidos em uma ocupação complexa e muitas vezes estressante (Smith \& Moss, 2009). Sua prática é permeada por demandas emocionais intensas, por vezes excessivas, que exigem uma relação próxima com as pessoas, o que torna estes profissionais suscetíveis aos estressores ocupacionais (Moreno-Jimenez, Meda-Lara, MoranteBenadero, Rodrígue-Munõz \& PalomeraChávez, 2006).

A maior parte dos eventos estressantes que as pessoas lidam cotidianamente são do tipo relacional (Folkman, 2009). 0 processo relacional é inerente $\mathrm{e}$ componente transversal à atividade do Psicólogo, independente do seu campo de atuação (Rocha, Silva, Barbosa, Duarte, 2013). Moore e Cooper (1996) pontuam que os profissionais de saúde mental enfrentam diariamente tensão emocional adicional pela própria natureza de suas profissões em lidar com pessoas com problemas, muitas vezes durante longos períodos de tempo.

O principais estressores no trabalho do psicólogo apontados na literatura são a preocupação constante com pacientes e clientes, mesmo fora do contexto de trabalho (Grafanaki et al., 2005; Sherman \& Thelen, 1998), a diminuição do controle individual sobre o trabalho, a longa duração da jornada de trabalho, o aumento do tempo envolvido em atividades burocráticas (Rupert \& Morgan, 2005) e o atendimento às expectativas de ser um modelo de saúde mental e lidar eficazmente com os mais diferentes estressores e dificuldades (Sherman, 1996).

Assim, considerando que a profissão de psicólogo tem um viés de gênero feminino (Castro \& Yamamoto, 1998), possui atribuições de cunho predominantemente relacional e assistencial e que esse profissional se encontra diante de um novo cenário de transformações que tem demandado diferentes exigências do trabalho (Bastos, Gondim, \& BorgesAndrade, 2011; Cruz \& Schultz, 2009), justifica-se a relevância do presente estudo. Sua realização também permite a produção de conhecimento útil, contribuindo diretamente para o campo da psicologia da saúde ocupacional em uma categoria profissional ainda pouco estudada. Pelo exposto, o presente estudo teve como objetivo avaliar as estratégias de enfrentamento utilizadas por psicólogos frente a estressores ocupacionais e identificar diferenças de acordo com o sexo a partir de uma perspectiva de gênero. 
MÉTODO

Participantes

A amostra foi constituída por 518 psicólogos que atuam há mais de um ano no estado do Rio Grande do Sul. A maioria é composta de mulheres $(77,6 \%)$, com união estável $(60,2 \%)$, que não possuem filhos $(57,5 \%)$. A idade dos participantes variou de 23 a 65 anos $(M=34,7 ; D P=8,4)$. Em média, eles trabalham na profissão há 10 anos $(\mathrm{DP}=8,9)$, o tempo de atuação variando de 01 a 40 anos. A maioria trabalha exclusivamente na profissão $(74,1 \%)$, em uma única área $(58,1 \%)$ e com remuneração entre 3 a 6 salários mínimos $(51,6 \%)$.

\section{Instrumentos}

Os dados foram coletados por meio dos seguintes instrumentos autoaplicáveis: 1. Questionário de dados sociodemográficos (sexo, idade, estado civil, relação conjugal, filhos, faixa salarial) e laborais (tipo de vínculo empregatício, tempo de formação, tempo de experiência profissional, áreas de atuação, carga horária semanal); 2. Escala COPE de Carver, Sheier e Weintraub (1989), que avalia as estratégias de enfrentamento, sendo composta de 60 itens que dão origem a 15 fatores: o coping ativo, o planejamento, a supressão de atividades concomitantes, o coping moderado, a busca de suporte social por razões instrumentais, a busca de suporte social por razões emocionais, a reinterpretação positiva, a aceitação, o retorno para a religiosidade, 0 foco na expressão das emoções, a negação, $\quad 0 \quad$ comportamento descomprometido, 0 desengajamento mental, o humor e o uso de substâncias. A escala é avaliada por meio de um sistema de pontuação com variação de 1 a 4, sendo o 1 para "não costumo fazer isso nunca", o 2 para "costumo fazer isso um pouco", o 3 para "costumo fazer isso moderadamente" e o 4 para " costumo fazer isso muito".

\section{Procedimentos}

Os dados do estudo foram coletados por meio de pesquisa on-line e participação voluntária dos psicólogos. Para fins de recrutamento dos participantes, foi utilizada a técnica do Respondent Driven Sampling (RDS). Assim, os primeiros participantes ( $1^{\text {a }}$ onda) foram psicólogos que tiveram seus emails mapeados de sites de associações, Conselho Regional de Psicologia, sindicato e demais órgãos representativos da Psicologia. Uma ( $2^{a}$ onda) foi obtida por meio da divulgação do Conselho Regional de Psicologia e em duas redes sociais de profissionais dos quais as pesquisadoras fazem parte. Ainda, uma ( $3^{a}$ onda) foi obtida por meio do mapeamento de guias telefônicos virtuais (Goel \& Salganik, 2009; Heckathorn, 1997).

Os psicólogos receberam um e-mail convite para a participação na pesquisa e aqueles que 0 aceitavam assinalaram com um " $X$ " o Termo de Consentimento Livre e Esclarecido, respeitando-se, assim, as diretrizes éticas da Resolução 466 do Conselho Nacional de Saúde no tocante à realização de pesquisa com seres humanos (Ministério da Saúde, 2012). O estudo foi aprovado pelo Comitê de Ética em Pesquisa da PUC/RS sob o Parecer $n^{0} 445847$.

Quanto a análise dos dados, foram realizadas análises descritivas de caráter exploratório para avaliar a qualidade do banco de dados, verificando possíveis erros de digitação, distribuição de itens, casos omissos e extremos. Para a análise das estratégias de enfrentamento os 15 fatores foram agrupados em três estratégias de enfrentamento: coping focado no problema (coping ativo, planejamento, supressão de atividades concomitantes e busca de suporte social por razões instrumentais); coping focado na emoção (coping moderado, busca de suporte social por razões emocionais, reinterpretação positiva e crescimento, aceitação, retorno para a religiosidade, foco na emoção e humor) e 
coping de evitação (negação, comportamento descomprometido, desengajamento mental e uso de substâncias) (Carver \& Scheier, 1989; Dobreva-Martinova, Villeneuve, Strickland, \& Matherson, 2002; Litman, 2006).

A comparação dos grupos foi realizada por meio da prova $t$ de student e do teste Qui-quadrado, adotando-se como significativo $o$ valor de $p \leq 0,05$. 0 tamanho do efeito foi calculado pela diferença média padronizada entre dois grupos (d de
Cohen), que considera 0,20 um valor indicativo de um efeito pequeno, 0,50 um efeito médio e 0,80 um tamanho de efeito grande.

\section{RESULTADOS}

A análise comparativa das características sociodemográficas e laborais não evidenciou diferenças entre os participantes homens e mulheres, de acordo com a tabela 1 e 2 .

TABELA 1.

Perfil sociodemográfico e laboral das variáveis qualitativas de acordo com o sexo.

\begin{tabular}{lccccc}
\hline \multirow{2}{*}{ Variáveis } & \multicolumn{3}{c}{ Homens $(\mathrm{n}=187)$} & \multicolumn{3}{c}{ Mulheres $(\mathrm{n}=354)$} \\
\cline { 2 - 6 } & $\mathrm{n}$ & $\%$ & $\mathrm{n}$ & $\%$ & $\mathrm{p}$ \\
\hline Situação conjugal & & & & & 0.917 \\
Com união estável & 59 & $(60,8)$ & 124 & $(60,2)$ & \\
Sem união estável & 38 & $(39,2)$ & 82 & $(39,8)$ & \\
Filhos & & & & & \\
Não & 62 & $(53,4)$ & 236 & $(58,9)$ & 0.300 \\
Sim & 54 & $(46,6)$ & 165 & $(41,1)$ & \\
Remuneração & & & & & \\
Até 3 SM & 36 & $(31,3)$ & 91 & $(22,7)$ & 0.051 \\
3 SM a 6 SM & 60 & $(52,2)$ & 206 & $(51,4)$ & \\
Superior a 6 SM & 19 & $(16,5)$ & 104 & $(25,9)$ & \\
\hline
\end{tabular}

TABELA 2.

Perfil sociodemográfico e laboral das variáveis quantitativas de acordo com o sexo.

\begin{tabular}{llllll}
\hline Variáveis & Sexo & $\mathrm{N}$ & $\mathrm{M}$ & $\mathrm{DP}$ & $p$ \\
\hline \multirow{2}{*}{ Idade } & Fem & 400 & 34,12 & 7,07 & 0,061 \\
& Masc & 116 & 35,92 & 9,09 & \\
\multirow{2}{*}{ Tempo de atuação profissional } & Fem & 401 & 10,69 & 8,20 & 0,697 \\
& Masc & 116 & 10,34 & 8,60 & \\
\multirow{2}{*}{ Tempo formação Profissional } & Fem & 400 & 10,85 & 8,47 & 0,735 \\
& Masc & 115 & 10,55 & 8,57 & \\
Carga horária semanal & Fem & 400 & 33,45 & 13,07 & 0,303 \\
& Masc & 116 & 32,03 & 13,24 & \\
\hline
\end{tabular}

Os resultados revelam que as estratégias de coping mais utilizadas pelos psicólogos que compuseram a amostra foram as focadas no problema (Tabela 3 ).

TABELA 3.

Amplitude, médias, desvios padrão e alfas das estratégias de enfrentamento.

\begin{tabular}{lccccc}
\hline Estratégias de enfrentamento & Min & Max & M & DP & a \\
\hline Coping focado no problema & 1,50 & 4,00 & 2,92 & 0,38 & 0,77 \\
Coping focado na emoção & 1,18 & 4,00 & 2,53 & 0,32 & 0,80 \\
Coping de evitação & 1,00 & 4,00 & 1,75 & 0,46 & 0,87 \\
\hline
\end{tabular}


$\mathrm{Na}$ análise, considerando a variável sexo, a única diferença encontrada é que as mulheres apresentam médias mais elevadas nas estratégias focadas na emoção. (Tabela 4). O valor obtido por meio do 0,32 indicando um médio poder de efeito.

TABELA 4.

Comparação de médias das estratégias de enfrentamento de acordo com sexo.

\begin{tabular}{llccccc}
\hline Estratégias de enfrentamento & Sexo & N & M & DP & $p$ & $d$ \\
\hline \multirow{2}{*}{ Coping focado no problema } & Fem & 401 & 2,90 & 0,38 & 0,181 & \\
& Masc & 116 & 2,96 & 0,39 & & \\
\multirow{2}{*}{ Coping focado na emoção } & Fem & 401 & 2,55 & 0,32 & $0,007^{*}$ & 0,32 \\
& Masc & 116 & 2,45 & 0,31 & & \\
\multirow{2}{*}{ Coping de evitação } & Fem & 401 & 1,73 & 0,46 & 0,068 & \\
\hline Nota: $\left.{ }^{*}\right) p<0,05 ; d=$ Effect size Cohen's d & Masc & 116 & 1,83 & 0,46 & & \\
\hline
\end{tabular}

\section{DISCUSSÃO}

0 presente estudo buscou avaliar as estratégias de enfrentamento utilizadas por psicólogos frente a estressores ocupacionais e identificar diferenças de acordo com o sexo. $\mathrm{Na}$ análise descritiva, considerando a totalidade da amostra, verifica-se uma maior utilização das estratégias de coping focadas no problema (coping ativo, planejamento, supressão de atividades concomitantes e busca de suporte social por razões instrumentais), de acordo com o modelo de Carver, Scheier e Weintraub (1989). Este resultado pode ser compreendido a partir das especificidades da formação do psicólogo, baseada no modelo de supervisão da atuação profissional. Tal prática é fomentada desde a graduação e, geralmente, se estende para a vida profissional (Falender et al. 2004; Falender \& Shafranske, 2007; Roe, 2003). É comum o profissional manter uma rede de trocas através de grupos de estudo e de supervisão realizada por um profissional com maior experiência. O modelo de formação profissional do psicólogo, segundo Roe (2003) envolve o desenvolvimento de competências básicas que são efetivadas no curso de graduação, competências iniciais desenvolvidas na fase de transição profissional por meio de prática supervisionada, geralmente no primeiro ano de experiência profissional, e de competências avançadas, que se consolidam nos anos seguintes, geralmente em 4 ou 5 anos após a formação.

A formação em Psicologia estabelece uma cultura de valorização da autoavaliação e prática reflexiva mediada pelo feedback de professores, orientadores e colegas. Esta formação enfatiza, ainda, 0 desenvolvimento de capacidades para analisar questões geradoras de tensão e desconforto para qualificar seu desempenho (Kaslow et al., 2007). Nesse contexto entende-se 0 maior uso das estratégias focadas no problema, no sentido de buscar ajuda e informações sobre o estressor, pensar e planejar ações estabelecendo passos sucessivos para remover, atenuar ou melhorar os efeitos do mesmo.

Este possível entendimento corrobora os resultados obtidos em estudo de Teixeira, Furtado, Poça, Rossini e Caggiano (2003) com estudantes de psicologia. Nesse, alunos de início de curso utilizavam com maior frequência estratégias de fuga e esquiva frente a estressores relacionados a mudanças de vida, enquanto estudantes de final do curso utilizavam com maior frequência estratégias voltadas para a resolução de problemas. Pode-se supor que as habilidades desenvolvidas no decorrer do curso auxiliem a adoção de estratégias mais 
adaptativas. $O$ presente resultado alinha-se igualmente ao encontrado em estudo realizado por Cushway e Tyler (1994) com 101 psicólogos estadunidenses, no qual a busca de suporte social instrumental foi apontada como a estratégia mais utilizada.

$\mathrm{Na}$ análise de acordo com o sexo dos participantes verificou-se que mulheres adotam com maior frequência estratégias focadas na emoção (coping moderado, busca de suporte social por razões emocionais, reinterpretação positiva e crescimento, aceitação, retorno para a religiosidade, foco na emoção e humor). 0 mesmo resultado foi identificado em estudos de Soderstrom et al. (2000) e Matud (2004).

Estudo realizado por Matud (2004) identificou que mulheres classificam os estressores de forma mais negativa $e$ menos controláveis do que os homens. As mulheres pontuaram significativamente mais alto do que os homens no uso de estratégias emocionais e mais baixo nas estratégias racionais. Em contrapartida, os homens apresentaram maior inibição emocional do que as mulheres. Estudo de meta-análise realizado por Tamres, Janicki, e Helgeson (2002), a fim de examinar a diferença de gênero no uso de estratégias de enfrentamento, identificou maior propensão das mulheres para expressar-se verbalmente, buscar apoio social emocional e ruminar problemas para si e para os outros.

$\mathrm{Na}$ presente amostra, as diferenças de gênero identificadas podem possivelmente ser entendidas através da investigação dos processos de socialização de homens e mulheres, uma vez que não foram identificadas diferenças significativas nas variáveis sociodemográficas e laborais entre os grupos analisados. Enquanto socialmente às mulheres atribui-se uma suposta facilidade para 0 manejo de emoções e sentimentos (Purvanova \& Muros, 2010; Rohlfs, 1999), aos homens são conferidas características como praticidade e objetividade (Caetano \& Neves, 2009). Pode-se conjecturar que tais características impliquem em um maior distanciamento emocional. A socialização diferenciada entre os sexos reserva à mulher um papel expressivo (orientado para o contato interpessoal) e ao homem um papel instrumental (orientado para metas), com impacto sobre as escolhas e desempenho profissional (Rosemberg, 1984).

Marcondes (2013) enfatiza o cuidado de pessoas como elemento estrutural do feminino. A afirmação do cuidado como estruturante da identidade feminina justifica, em parte, as escolhas profissionais femininas ligadas à atenção dos outros.

Destaca-se a necessidade de aprofundamento da discussão dos resultados obtidos, tendo em vista que os mesmos não podem ser generalizados, pois se trata de uma amostra não probabilística. Também é importante destacar que 0 tamanho do efeito das diferenças encontradas foi médio, indicando que os resultados podem ser semelhantes na população de psicólogos.

Assim, é importante dar seguimento à realização de novos estudos, a partir de amostras probabilísticas com amostra nacional de psicólogos, a fim de ampliar a compreensão sobre as estratégias de enfrentamento nesta categoria profissional e sua relação com gênero. Tais medidas possibilitariam uma melhor comparação dos resultados, pois a literatura sobre estratégias de enfrentamento utilizadas por psicólogos ainda é incipiente.

Analisar as variações de gênero com base nas estratégias de enfrentamento pode oferecer informações importantes para a qualificação e desenvolvimento de políticas educacionais e formativas, assim como para a prevenção de adoecimento ocupacional e desenvolvimento de programas de saúde que atendam às 
necessidades diferenciadas de homens e mulheres (Fielden \& Davidson, 2001).

A natureza predominantemente relacional da atividade do psicólogo exige alta demanda emocional (Moreno-Jiménez et al., 2006; Rupert, Stevanovic \& Hunley, 2009), 0 que 0 torna vulnerável ao desenvolvimento de adoecimento ocupacional, particularmente a Síndrome de Burnout, tipo de estresse ocupacional de caráter crônico que atinge profissionais envolvidos com o cuidado de outros (Smith \& Moss, 2009). Desenvolver 0 conhecimento e a compreensão das estratégias de enfrentamento de psicólogos pode proporcionar um melhor conhecimento do seu repertório de estratégias de enfrentamento de estressores, cada vez mais presentes na profissão, visando 0 desenvolvimento de estratégias com foco na resolução de problemas, uma vez que são essas que proporcionam melhores indicadores de saúde e qualidade de vida e previnem a ocorrência de possíveis doenças ocupacionais.

\section{REFERÊNCIAS}

Bastos, A. V. B., Gondim, S. M. G., \& Borges-Andrade, J. E. (2011). As mudanças no exercício profissional da psicologia no Brasil: o que se alterou nas duas últimas décadas e o que vislumbramos a partir de agora? In A. V. B. Bastos \& S. M. G. Gondin (Orgs.), O Trabalho do Psicólogo no Brasil (pp. 419-495). Porto Alegre: Artmed.

Caetano, E., \& Neves, C. E. (2009). Relações de gênero e precarização do trabalho docente. Revista HISTEDBR On-line, (n. especial), 251-263. Disponível em: http://www.histedbr.fae.unicamp.br/revi sta/edicoes/33e/art16_33esp.pdf.

Carver, C. S., Sheir, M. F., \& Weintraub, B. (1989). Assessing coping strategies: a theoretically based approach. Journal of Personality and Social Psychology, 56, 267-283.

Castro, A. E. F. de, \& Yamamoto, O. H. (1998). A Psicologia como profissão feminina: apontamentos para estudo. Estudos de Psicologia (Natal), 3(1), 147-158.

Collins, S. (2008). Statutory social workers: stress, job satisfaction, coping, social support and individual differences. British Journal of Social Work, 38(6), 1173-1193. doi: 10.1093/bjsw/bcm047

Cruz, R. M., \& Schultz, V. (2009). Avaliação de competências profissionais e formação de psicólogos. Arquivos Brasileiros de Psicologia, 61(3), 117127.

Cushway, D., \& Tyler, P. A. D. (1994). Stress and coping in clinical psychologists. Stress \& Health, 10(1), 35-42. doi: $10.1002 /$ smi.2460100107

Falender, C.I A., \& Shafranske, E. P (2007). Competence in competency-based supervision practice: Construct and application.Professional Psychology: Research and Practice, 38(3), 232-240. doi: 10.1037/0735-7028.38.3.232

Falender, C. A. et al ... Grus, C. (2004). Defining competencies in psychology 
supervision: A consensus statement. Journal of Clinical Psychology, 60(7), 771-785. doi: 10.1002/jclp.20013

Folkman, S., \& Lazarus, R. S. (1980). An analysis of coping in a middle-aged community sample. Journal of Health and Social Behavior, 21(3), 219-239.

Folkman, S. (2009). Questions, answers, issues, and next steps in stress and coping research. European Psychologist, 14(1), 72-77.

Gil-Monte, P. R. (2005). El síndrome de quemarse por el trabajo (burnout). Una enfermidad laboral en la sociedad del bienestar. Madri: Pirâmide.

Grafanaki, S., Pearson, D., Cini, F., Godula, D., McKenzie, B., Nason, S., et al. (2005). Sources of renewal: A qualitative study on the experience and role of leisure in the life of counselors and psychologists. Counselling Psychology Quarterly, 18, 31-40.

Graven, L. J., \& Grant, J. S. (2013). Coping and health-related quality of life in individuals with heart failure: An integrative review. Heart \& Lung: The Journal of Acute and Critical Care, 42(3),183-194.

doi:10.1016/j.hrtlng.2012.12.002

Heckathorn, D. D. (1997). Respondentdriven sampling: a new approach to the study of hidden populations. Social Problems, 44(2), 174-199.

Kaslow, N. J., Rubin, N. J., Forrest, L., Elman, N. S., Van Horne, B. A., Jacobs, S.C., et al. (2007). Recognizing, assessing, and intervening with problems of professional competence. Professional Psychology: Research and Practice, 38, 479-492.

Keogh, E., \& Herdenfeldt, M. (2002). Gender, coping and the perception of pain, Pain, 97(3), 195-201.

Lassance, M. C. P., \& Magalhães, M. 0. (1997). Gênero e escolha profissional. In R. S. Levenfus (Org.), Psicodinâmica da escolha profissional (pp. 47-61). Porto Alegre: Artes Médicas

Marcondes, M. M. (2013). O cuidado na perspectiva da divisão sexual do trabalho: contribuições para os estudos sobre a feminização do mundo do trabalho. In Yamoullas, S. C. Trabalhadoras: análise da feminização das profissões e ocupações (pp. 251280). Brasília: Abaré Editorial.

Matud, M. P. (2004). Gender differences in stress and coping styles. Personality and Individual Differences, 37(7), 14011415. doi: 10.1016/j.paid.2004.01.010

Mazzola, J. J., Schonfeld, I. S., \& Spector, P. E. (2011). What qualitative research has taught us about occupational stress, $\quad 27(2), \quad 93-110$. doi: $10.1002 / s m i .1386$

Moore, K. A., \& Cooper, C. L. (1996). Stress in mental health professionals: A theoretical overview. International Journal of Social Psychiatry, 42, 82-89.

Nolen-Hoeksema, S. (2011). Emotion Regulation and Psychopathology: The Role of Gender. Annual Review of Clinical Psychology, 8, 161-187. doi: 10.1146/annurev-clinpsy-032511143109

Piko, B. (2001). Gender differences and similarities in adolescents' ways of coping. The Psychological Record, 51, 223-235.

Puranova, R. K., \& Muros, J. P. (2010). Gender differences in burnout: a metaanalysis. Journal of Vocational Behavior, 77, 168-185. Disponível em: http://www.sciencedirect.com/science/a rticle/pii/S0001879110000771.

Ptacek, J. T., Smith, R. E., \& Dodge, K. L. (1994). Gender differences in coping with stress: when stressor and appraisals do not differ. Personality and Social Psychology Bulletin, 20, 421-430.

Rohlfs, D. 1999. La perspectiva de género en el estudio de las diferencias y desigualdades en salud. I Jornada de la Red de Médicas y Profesionales de la Salud, 2, 12-13.

Rocha, A. C., Silva, G., Barbosa, R., \& Duarte, C. (2013). Tornar-se Psicólogo para além das aulas: grupo de desenvolvimento com estudantes de 
Psicologia. Análise Psicológica, 31(1), 87-102.

Roe, R. (2003). What makes a competent psychologist?.

European

Psychologist, 7(3), 192-202.

Rosemberg, F. (1984). Afinal, por que somos tantas psicólogas?. Psicologia: Ciência e Profissão, 4(1), 6-12.

Rupert, P. A., \& Morgan, D. J. (2005). Work setting and burnout among professional psychologists.

Professional

Psychology: Research and Practice, 36, $544-550$.

Sherman, M. D. (1996). Distress and professional impairment due to mental health problems among psychotherapists. Clinical Psychology Review, 16, 299-315.

Sherman, M. D., \& Thelen, M. H. (1998). Distress and professional impairment among psychologists in clinical practice. Professional Psychology: Research and Practice, 29, 79-85.

Smith, P. L., \& Moss, B. (2009). Psychologist impairment: what is it, how can it be prevented, and what can be done to address it? Clinical Psychology: Science and Practice, 16(1), 1-15.

Stevanovic, P., \& Rupert, P. A. (2004). Career-sustaining

satisfactions, and stresses of professional psychologists.

Psychotherapy: Theory, Research, Practice, Training, 41, 301-309.

Tamres, L. K., Janicki, D., \& Helgeson, V. S. (2002). Sex differences in coping behavior: a meta-analytic review and an examination of relative coping. Personality and Social Psychology Review, 6, 2-30.

Teixeira, M. C. T. V., Furtado, R. M. G., Poça, S. R. da S., Rossini, A. C., \& Caggiano, J. O. (2003). Estratégias de enfrentamento a mudanças de vida em alunos de psicologia. Revista de Enfermagem da UERJ, 11(1) 39-46.

Watson, S. B., Goh, Y.W., \& Sawang, S. (2011). Gender influences on the workrelated stress-coping process. Journal of Individual Differences, 32(1), 39-46.

doi: 10.1027/1614-0001/a000033 